RESEARCH NOTE

\section{Ultrastructure of the} Adhesion of Crithidia guilhermei (Kinetoplastida: Trypanosomatidae) to Plastic Coverslips: Formation of a Fibrous Matrix Around the Cells

\section{Leila MF Oliveira, Maurilio J Soares*, Reginaldo P Brazil ${ }^{+}$, Wanderley de Souza**}

Laboratório de Entomologia Médica **Laboratório de Ultra-estrutura Celular, Departamento de Parasitologia e Biofísica Celular, Instituto de Biofísica Carlos Chagas Filho, UFRJ, 21949-000 Rio de Janeiro, RJ, Brasil *Departamento de Ultra-estrutura e Biologia Celular, Instituto Oswaldo Cruz, 21045-900 Rio de Janeiro, RJ, Brasil

Key words: Trypanosomatidae - Crithidia - adhesion ultrastructure

Crithidia guilhermei Soares, Brazil, Tanuri \& De Souza, 1986 is a trypanosomatid isolated from the hindgut of the fly Phaenicia cuprina by RP Brazil et al. (1984 Mem Inst Oswaldo Cruz 80 Suppl.: BI-52). It can be distinguished from other members of the Crithidia genus due to the presence of some alterations in the cell morphology, such as absence of the flagellar paraxial rod and branching of the tubular mitochondrion, which are typical remarks of endosymbiont-bearing trypanosomatids (E Freymuller \& EP Camargo 1981 J Protozool 28: 175-182). However, no symbionts are present in $C$. guilhermei (M Soares et al. 1986 Can J Zool 64: 2837-2842). Adhesion of parasites to inert substrates, such as Millipore filters (BE Brooker 1971 Protoplasma 72: 19-25)

\footnotetext{
This work has been supported by CNPq and FINEP.

${ }^{+}$Corresponding author. Present address: Laboratório de Leishmanioses, Centro de Pesquisas René RachouFIOCRUZ, Av. Augusto de Lima 1715, 30190-002 Belo Horizonte, MG, Brasil. Fax: +55-31-295.3115

Received 10 October 1996

Accepted 10 April 1997
}

chitosan (a chitin derivative) gels (KR Wallbanks et al. 1989 Acta Trop 46: 63-68, JR Stiles et al. 1990 Ann Trop Med Parasitol 84: 197-200) or plastic coverslips (M Hommel \& E Robertson 1976 Experientia 32: 464-466, S Maraghi et al. 1987 Ann Trop Med Parasitol 81: 457-458), mimicry the adhesion of the parasites to the midgut cells in the digestive tract of the invertebrate hosts, and therefore represents an in vitro model for the study of the parasite-host cell relationships, which however has not been fully explored. An in vitro study of the biophysical and biochemical mechanisms involved in the parasite-substrate attachment has summarized the research in this field (DH Molyneux et al. 1987 Trypanosomatid-vector interfaces. In vitro studies on parasite-substrate interaction, p. 387-396. In KP Chang \& D Snarry (eds) Host-Parasite and Molecular Interactions in Protozoal Infections, Springer-Verlag) .

In this study we analyzed some ultrastructural features of $C$. guilhermei adhered to plastic coverslips. We describe the presence of cross-bridges of a filamentous material between the adhered cells, which is similar to that found in vivo with Trypanosoma melophagium in the hindgut of the sheep ked, Melophagus ovinus (P Heywood \& DH Molyneux 1985 Cytobios 44: 183-188).

C. guilhermei has been maintained at $28^{\circ} \mathrm{C}$ with serial passages in Warren's liquid medium (LG Warren $1960 \mathrm{~J}$ Parasitol 46: 529-539) supplemented with $10 \%$ fetal calf serum. A plastic coverslip (Thermanox cover slips, Lux Sientific Co.) was laid into the culture tubes and the parasites were allowed to adhere to this substrate for $48 \mathrm{hr}$. The coverslips were then withdrawn from the tubes, washed for $5 \mathrm{~min}$ in phosphate buffered saline (PBS), pH 7.2, and processed microscopy-cally. The coverslips were cut in two and each half was then fixed for $1 \mathrm{hr}$ with $2.5 \%$ glutaraldehyde diluted in either $0.1 \mathrm{M}$ phosphate or cacodylate buffer, $\mathrm{pH}$ 7.2. Each half was then washed in the respective buffer, briefly post-fixed for $10 \mathrm{~min}$ with $1 \% \mathrm{OsO}_{4}$, washed in buffer, dehydrated in graded ethanol and critical point dried. The coverslips were then mounted on a stub, covered with a $5 \mathrm{~nm}$ thick layer of gold in a sputtering device and then observed by scanning electron microscopy (SEM). for Transmission electron microscopy (TEM), coverslips were cut in two and each half was fixed for 2 $\mathrm{hr}$ in $2.5 \%$ glutaraldehyde diluted in either $0.1 \mathrm{M}$ phosphate or cacodylate buffer, $\mathrm{pH}$ 7.2. Each half was then washed in the respective buffer, post-fixed for $1 \mathrm{hr}$ in $1 \% \mathrm{OsO}_{4}$, dehydrated in acetone and embedded in Epon. For the ultrastructural detection of carbohydrates, some coverslips were processed as described above, but $0.5 \mathrm{mg} / \mathrm{ml}(\mathrm{w} / \mathrm{v})$ ruthenium red was added to the fixative and to the 
washing buffers (JH Luft 1971 Anat Rec 171: 347368). The periodic acid-thiosemi-carbazide-silver proteinate technique (JP Thiéry 1967 J Microscopie 6: 987-1018) was also used for the detection of polysaccharides. Briefly, ultra-thin sections were collected on gold grids, incubated for $30 \mathrm{~min}$ in $1 \%$ periodic acid, washed in water and then incubated for $72 \mathrm{hr}$ in $1 \%$ thiosemicarbazide. The grids were then washed in $10 \%, 5 \%$ and $2 \%$ acetic acid, rinsed in distilled water and incubated for $30 \mathrm{~min}$ in the dark with $1 \%$ silver proteinate. After this last incubation, the grids were washed in distilled water and observed unstained by TEM. In control experiments, periodic acid treatment was omitted.

Previous experiments showed that culture forms (choanomastigotes) of $C$. guilhermei did not expontaneously adhere to glass coverslips in sufficient number to be studied in situ by electron microscopy. Coating of the glass coverslips with poly-L-lysine (M.W. 70,000) resulted in adhesion of the cells due to charge attraction, but examination of the coverslips by SEM showed that the parasites were attached to the substrate through the whole body and flagellum. However, introduction of plastic coverslips into the culture tubes resulted in attachment and colonization of this substrate by the protozoa. SEM showed that the parasites were adhered through the flagellum, either isolated or forming aggregates of several individuals. In isolated cells, the whole flagellum was usually sticked to the substrate, presenting occasion- ally lateral membrane expansions (Fig. 1). In the aggregates, several individuals were tightly arranged side by side in an upright position, and were adhered to the substrate through the tip of the flagellum. The aggregates of cells showed no typical morphological aspect: colonies could be rounded, although elongated strips were usually found (Fig. 2). Closer examination showed that filamentous bridges could be observed linking the cells (Fig. 3), independently of the fixative solution used.

TEM showed that the protozoan structure was not well preserved when cacodylate buffer was used. Therefore, only parasites processed with phosphate buffer were analyzed in more detail. The organisms were bound to the plastic coverslip by means of a hemidesmosome formed between the tip of the flagellum and the substrate (Fig. 4). The flagellum could be short or long, followed by the cell body, which usually made no contact with the plastic. Some cells could be observed very close to the plastic coverslip, but no specialized attachment structures could be seen between the plasma membrane and the substrate. A fibrous matrix was observed among the parasites, but not above the layer of cells. It consisted of thin filaments, about $9.5 \mathrm{~nm}$ (range: $7.0-13.0 \mathrm{~nm}$ ) in diameter. In adjoining parasites, most filaments formed crossbridges between the cells (Fig. 5). Filaments were present around cells processed with either cacodylate or phosphate buffer, despite the bad fixation

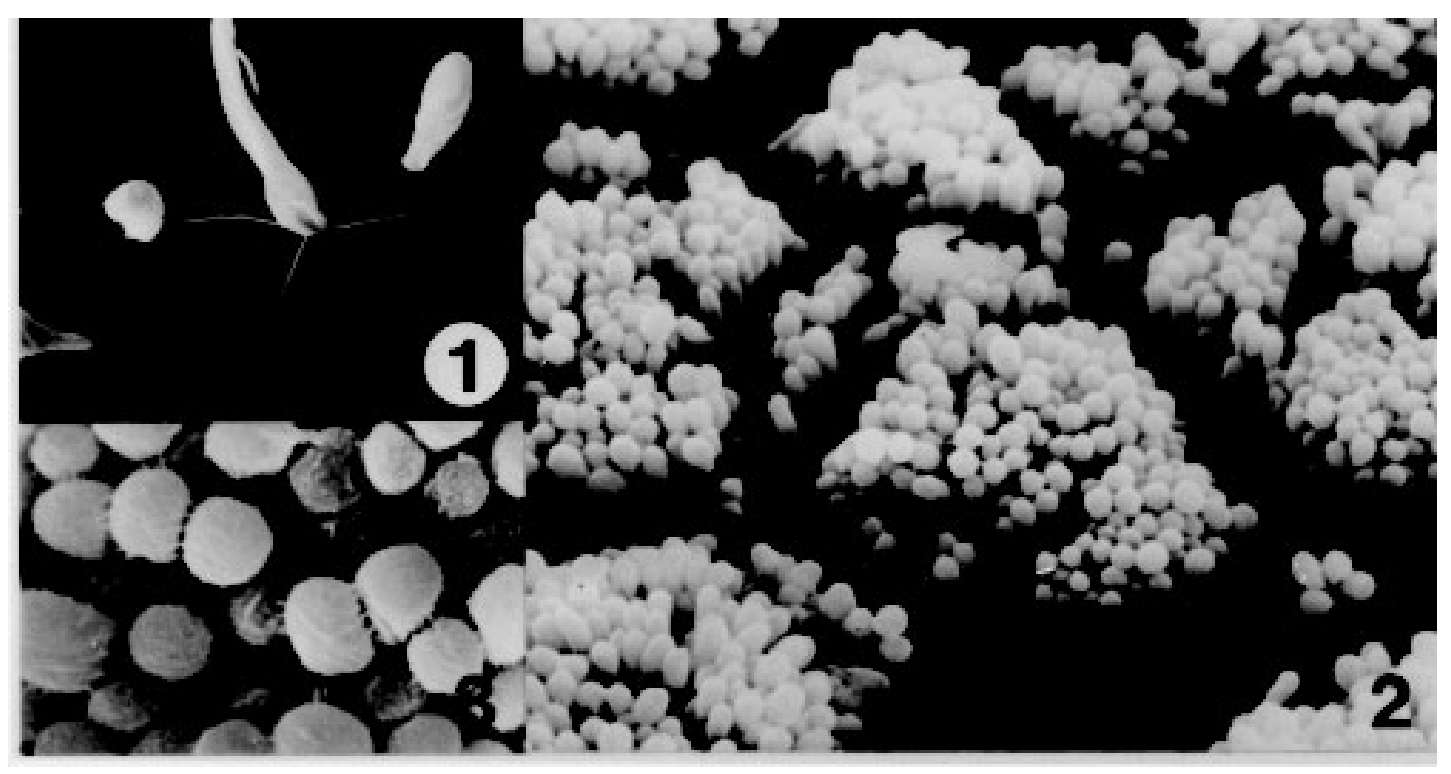

Figs 1 to 3 - Adhesion of Crithidia guilhermei to plastic coverslips. Scanning electron microscopy. Fig. 1: single cell, showing the flagellar adhesion to the substrate. Note expansions of the flagellar membrane. X 2,600. Fig. 2: an overview of several aggregates of adhered parasites. Each aggregate may contain more than a hundred individuals. X 1,400. Fig. 3: high magnification of a group of cells. Cross-bridges of filamentous material are seen among many organisms. X 4,500. 
obtained with the former buffer.

In cells processed in the presence of $0.5 \mathrm{mg} /$ $\mathrm{ml}$ ruthenium red for the detection of carbohydrates, positive staining of the filaments was observed (Fig. 6). Incubation of ultra-thin sections according to the Thiéry's technique for the detection of mucopolysaccharides also resulted in positive staining of the plasma membrane and of the extracellular filamentous matrix. Inside the cell, positive reaction was observed in the Golgi com- plex and in a tubular network found near the flagellar pocket region (Fig. 7). Reaction product was not observed in control sections processed without previous incubation in periodic acid.

It has been shown that parasites of the genera Trypanosoma and Leishmania adhere to the inner surface of several organs of the invertebrate hosts (insects) for division and differentiation ( $\mathrm{P}$ Thévenaz \& H Hecker 1980 Acta Trop 37: 163175, R Zeledón et al. 1984 Acta Trop 41: 39-43, L
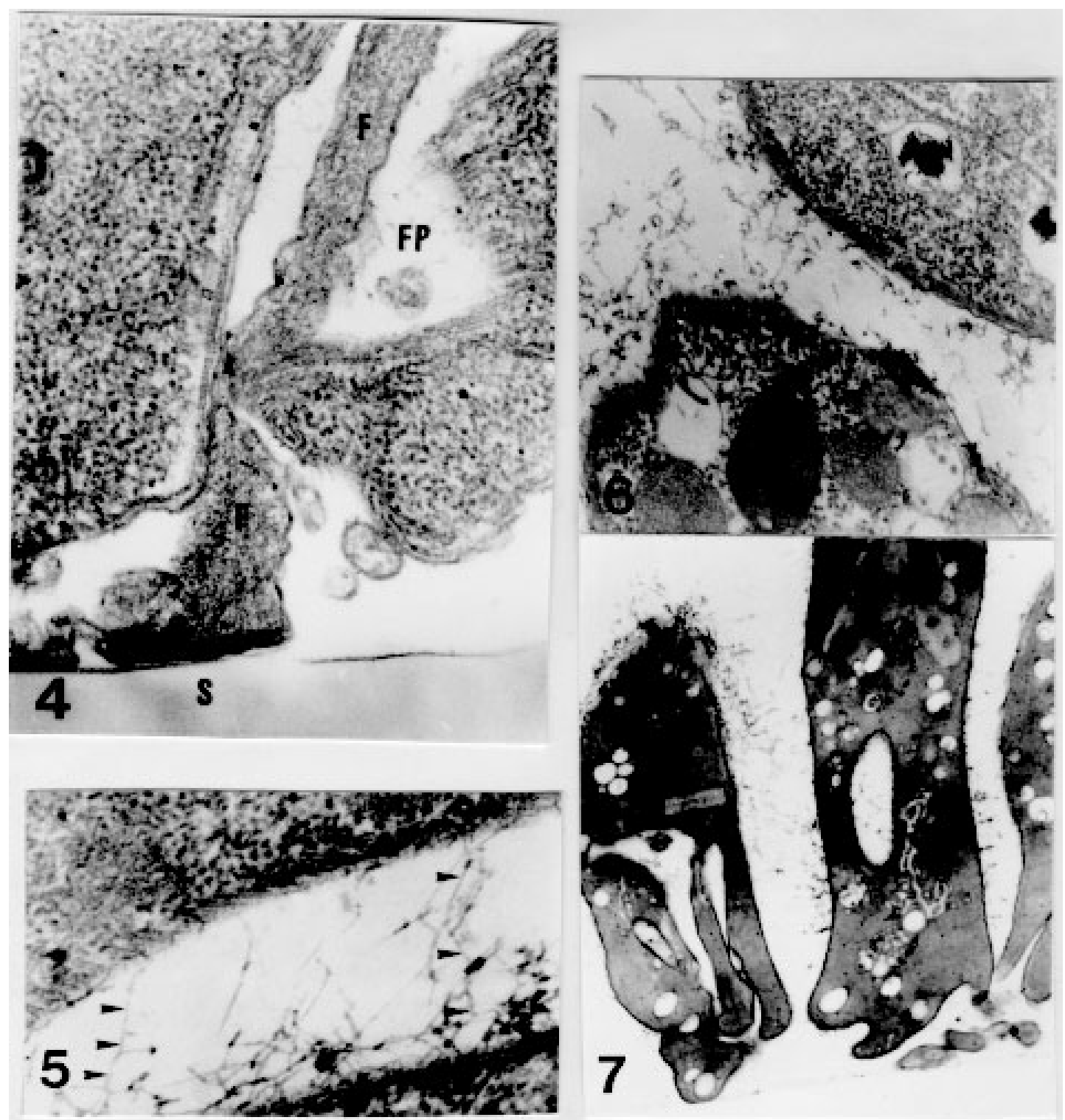

Figs 4 to 7 - Adhesion of Crithidia guilhermei to plastic coverslips. Transmission electron microscopy. Fig. 4: attachment of parasites to the plastic is mediated through hemidesmosomes formed between the tip of the flagellum $(\mathrm{F})$ and the inert substrate $(\mathrm{S})$. Note the presence of filaments inside the flagellar pocket (FP). X 60,000. Fig. 5: detail of the filamentous matrix. Some filaments form cross-bridges between the two adjacent cells (arrowheads). X 60,000. Fig. 6: cells incubated with ruthenium red in the presence of phosphate buffer. Electron-dense reaction product is seen between the cells. X 35,000. Fig. 7: ultra-thin sections incubated according to the Thiéry's technique for the detection of polysaccharides. Positive reaction was observed on the cell surface, as well as in the extracellular filamentous network. X 12,500. 
Tetley \& K Vickerman 1985 J Cell Sci 74: 1-19, KAK Hendry \& K Vickerman 1988 Parasitol Res 74: 403-408, LL Walters et al. 1987 Am J Trop Med Hyg 36: 294-314). However, even in monogenetic trypanosomatids attachment to the substrate it seems to be an essential step for the establishment of infections and survival of parasites. Adhesion is mediated through the flagellum, usually in the form of hemidesmosomes (BE Brooker 1976 $J$ Parasitol 72: 259-267) formed between the tip of the flagellum and the substrate, although other flagellar specializations have been described (reviewed by DH Molyneux 1977 Adv Parasitol 15: 1-82). Due to the importance of adhesion in the life cycle of trypanosomatids, the development of in vitro systems would allow detailed and reproducible studies on the parasite-substrate relationships (Molyneux et al. loc. cit.). Our data on C. guilhermei-plastic substrate bonding demonstrates that in vitro attachment can be successfully attained with monogenetic parasites, with multiplication of the cells. The few data obtained on adhesion of some trypanosomatids to inert substrates such as Millipore filters (Brooker 1971 loc. cit.), plastic (Hommel \& Robertson loc. cit., Maraghi et al. loc. cit.), bead-bound organic dyes (WR Fish et al. 1987 J Protozool 34: 457464 ) or chitin derivatives (Stiles et al. loc. cit., Wallbanks et al. loc. cit.) show that hemidesmosomes are formed at the tip of the flagellum, as occurs in vivo in the invertebrate hosts (reviewed in Molyneux et al. loc. cit.) It has been also shown that $T$. cruzi adhesion to glass induces multiplication and differentiation from epimastigote to trypomastigote forms (MC Bonaldo et al. 1988 J Cell Biol 106: 1349-1358, SM Krassner et al. 1990 J Protozool 37: 128-132). Such in vitro systems have been recently used to study the molecules involved in the attachment of the flagella to the substrate (Molyneux et al. loc. cit., A Warburg et al. 1989 J Protozool 36: 613-617).
Our results demonstrated that a fibrous matrix was formed around the parasites adhered in vitro to plastic coverslips. Such fibrous matrix has been already observed in vivo in T. melophagium (Heywood \& Molineux loc. cit.) T. corvi (LM Mungomba et al. 1989 Parasitol Res 75: 167-174), and L. major (R Killick-Kendrick et al. 1988 Parasitol Res 74: 586-590, T Lang et al. 1991 Eur $J$ Cell Biol 55: 362-372). Detection of such filaments under in vitro conditions shows that this material is not of host origin, but is secreted by the parasites. Our findings of positive reaction for carbohydrates in the Golgi complex, flagellar pocket and the cell surface of $C$. guilhermei further suggests that this material is secreted, through the flagellar pocket, by the protozoa. Accordingly, it has been recently shown that in vitro cultured promastigotes (insect stages) of L. mexicana secrete an acid phosphatase (a phosphoglycoprotein), which appears as filaments on the surface of the cells (T Ilg et al. 1991 Proc Natl Acad Sci USA 88: 8774-8778). Positive reaction in the filaments after incubation of the parasites according to two different techniques for the detection of carbohydrates suggests that these filaments contain carbohydrates. In L. major, it has been suggested that lipophosphoglycan was the major carbohydrate component of this extracellular matrix (Lang et al. loc. cit.). Since treatment of T. microti and L. major with several enzymes (including some glucosidases) did not result in detachment of the parasites (Molyneux et al. loc. cit.), it is possible that these filaments are not directly linked to the adhesion of the trypanosomatids to the substrate. The presence of cross-bridges of filaments among several parasites (but not between parasites and the substrate) as observed in $C$. guilhermei suggests that these filamentous matrix may play a role in keeping the parasites together. In an in vivo condition, this matrix would provide a mechanical support to prevent parasites from being removed from the insect gut during a meal, as previously speculated by D Jefferies et al. (1986 Acta Trop 43: 43-53). 